\title{
Sleep Quality in Non Dialysis Chronic Kidney Disease: Associated Factors and Influence on Prognosis
}

\author{
Jesús Calviño ${ }^{1 *}$, Lourdes Gonzalez-Tabarés ${ }^{1}$, Secundino Cigarrán², Beatriz Millán1, \\ Monica Guijarro33, Carmen Cobelo1, Maria-Jesús Sobrido4,5 \\ ${ }^{1}$ Department of Nephrology, Hospital Lucus Augusti, EOXI Lugo-Cervo-Monforte, Lugo, Spain \\ ${ }^{2}$ Department of Nephrology, Hospital da Costa, EOXI Lugo-Cervo-Monforte, Burela, Lugo, Spain \\ ${ }^{3}$ Department of Neurology, Hospital Lucus Augusti, EOXI Lugo-Cervo-Monforte, Lugo, Spain \\ ${ }^{4}$ Department of Neurology, Fundación Pública Galega de Medicina Xenómica-SERGAS, Instituto de \\ Investigación Sanitaria, Santiago de Compostela, Spain \\ ${ }^{5}$ Department of Neurology, Hospital San Rafael, A Coruña, Spain \\ Email: "jesus.calvino.varela@sergas.es
}

Received 9 May 2016; accepted 12 July 2016; published 15 July 2016

Copyright (C) 2016 by authors and Scientific Research Publishing Inc.

This work is licensed under the Creative Commons Attribution International License (CC BY). http://creativecommons.org/licenses/by/4.0/

(c) (7) Open Access

\section{Abstract}

Deficient sleep quality (SQ) has been linked with a higher hospitalization rate and mortality in dialysis patients, however the prevalence of sleep disorders and their influence on prognosis in non-dialysis chronic kidney disease (CKD) has been poorly investigated. The aim of this study was to assess factors related with SQ in CKD patients (stages I-IV) followed in a nephrology outpatient clinic as well as the long-term impact of SQ on patient's outcome. Between January and May 2008, Pittsburgh Sleep Quality Index (PSQI) was self-administered by 122 patients (68 males and 54 females) with a mean age of 65 years. Patients were classified as "good" (global PSQI <6) and "poor" sleepers (global PSQI $\geq 6$ ). We identified $66(54 \%)$ poor sleepers (PS), characterized by an older age ( $66 \pm 14.2$ vs $57 \pm 17.0, p<0.01)$, female predominance $(59 \%$ vs $26 \%, p<0.01)$ and worse renal function ( $49 \pm 19.1$ vs $57 \pm 23.2 \mathrm{ml} / \mathrm{min}, \mathrm{p}<0.05)$. There was a significant correlation between phosphate and PSQI score $(r=0.234, p=0.01)$, however no correlation with calcium or PTH. Vitamin D was also lower in PS $(17 \pm 7.2$ vs $23 \pm 15.1 \mathrm{ng} / \mathrm{ml}, \mathrm{p}<0.05)$. Until June 2015 , hospitalization rate was higher among PS $(64 \%$ vs $44 \%, p<0.05)$. In this period, there was also a trend towards higher mortality for PS $(18 \%$ vs $16 \%)$. In summary, over $50 \%$ of CKD patients have poor SQ, which was associated with older age, female gender, worse renal function, lower vitamin $D$ and higher phosphate levels. Deficient sleep was associated with a greater probability of hospitalization and might be a prognostic marker in CKD.

\footnotetext{
${ }^{*}$ Corresponding author.
}

How to cite this paper: Calviño, J., Gonzalez-Tabarés, L., Cigarrán, S., Millán, B., Guijarro, M., Cobelo, C. and Sobrido, M.-J. (2016) Sleep Quality in Non Dialysis Chronic Kidney Disease: Associated Factors and Influence on Prognosis. International Journal of Clinical Medicine, 7, 481-486. http://dx.doi.org/10.4236/ijcm.2016.77053 
Keywords

Chronic Kidney Disease, Sleep Quality, Hospitalization, Pittsburgh Sleep Quality Index, Vitamin D

\section{Introduction}

Sleep impairment is frequent among dialysis patients (40\% - 80\%). Insomnia or insufficient sleep time, sleep latency and daytime sleepiness, restless legs syndrome (RLS) and obstructive sleep apnea (OSA) are the most common complaints [1] [2]. Factors related with sleep disturbances in these patients include those also associated with poor sleep in the general population, such as age, diabetes, depression, and obesity. Sleep disturbances in dialysis patients have also been related with uremia, anemia, phosphate and inflammation. The association between sleep quality (SQ) and renal disease may be bidirectional, since depressive symptoms and fluid overload that are common in chronic kidney disease (CKD), may also impair SQ. Besides affecting quality of life, reduced SQ has been linked with higher morbidity and mortality rates in dialysis patients, as well as with more frequent hospitalization [3].

In non-dialysis CKD, sleep disturbances may represent a novel risk factor contributing to renal and cardiovascular damage; thus, improving SQ could help to reduce progression of CKD and cardiovascular events in these patients [4]. Although a high prevalence of sleep disorders would be expected in CKD before renal replacement therapy, this question has not been investigated in depth. Sleep surveys carried out in this population were usually based in clinical questionnaires, without objective measures like polysomnography or even expert examination. For example, we've recently reported that RLS frequency (after expert examination) in non-dialysis CKD is not very different from that described in the general population [5]. Moreover, to the best of our knowledge the relationship between SQ in early stages of CKD and the use of healthcare services has not been examined.

Therefore, this study was carried out to investigate SQ in CKD patients (stages I to IV), the factors associated with poor sleep and the relationship between sleep disturbances, hospitalization and long-term patient's outcome.

\section{Methods}

We used a validated Spanish translation of the Pittsburgh Sleep Quality Index (PSQI), a self-rating questionnaire developed to evaluate SQ during the previous months. PSQI contains 19 questions (each scored 0 to 3 ) to assess seven components of SQ (subjective SQ, sleep latency, sleep duration, habitual sleep efficiency, sleep disturbances, use of sleeping medications and daytime dysfunction) [6]. The total PSQI score ranges from 0 to 21. A global score of 6 or greater indicates a poor sleeper, meaning that the person suffers from moderate sleep problems in at least three sleep component areas, or severe sleep problems in two areas.

Between January and May 2008, patients with CKD (stages I to IV) attending the nephrology outpatient clinic were asked to voluntary answer the PSQI questionnaire. Only those with a complete and properly filled questionnaire were included in the study. Subjects under 18 years old, pregnant women and renal transplant patients were not included. The final group comprised 122 patients (68 men and 54 women) with a median age of $62 \pm$ 16.2 years. According to PSQI, patients were divided into "poor sleepers" [(PS); PSQI $\geq 6$ ] and "good sleepers" [(GS); PSQI < 6]. Seven years later (June 2015), the hospitalization episodes, renal function outcome and mortality rate were analyzed through retrospective evaluation of the participants' electronic medical records. The following clinical and demographic characteristics were obtained: 1) Presence of cardiovascular disease (defined as the presence of ischemic cardiomyopathy, stroke and/or peripheral arteriopathy that needed by-pass or amputation), chronic obstructive pulmonary disease (COPD), depression (defined by the need of antidepressant treatment) and diabetes; 2) Medications that might influence on symptoms, including antidepressants and hypnotic drugs. Body mass index (BMI) was calculated by the usual formula $\left(\mathrm{Kg} / \mathrm{m}^{2}\right)$. Laboratory parameters were obtained from routine blood and urine samples, including serum creatinine, uric acid, proteinuria (albumin to creatinine ratio), calcium, phosphorus, parathormone, vitamin D, C-reactive protein (CRP), hemoglobin, folate and iron status. Glomerular filtration rate (eGFR) was estimated by the four-variable Modification of Diet in Renal Disease (MDRD-4) equation [7]. 
The statistical package for social sciences software (SPSS version 15.0) was used for statistical analysis of differences between GS and PS. Results were expressed as mean and standard deviation or number and percent, as appropriate. The coefficient of skewness and the coefficient of kurtosis were used to evaluate normal distribution of the data. Continuous variables were compared using the Student's t-test or Mann-Whitney $U$-test. The bivariate correlation test was performed to determine the association of demographic and biochemical data with the PSQI score, using Pearson's correlation test and Spearman's correlation test for normally and non-normally distributed variables, respectively. Chi-square test and Fisher's exact test, as appropriate, were used to compare categorical variables. The level of significance was set at $\mathrm{p}<0.05$. Multiple logistic regression was applied for factors that reached statistical significance in order to identify variables independently associated with SQ.

\section{Results}

Out of 122 studied patients, 39 (32\%) were on CKD stages I-II, 65 (53\%) on stage III and 18 (15\%) on stage IV following the K/DOQI staging system [8]. 38 patients (31\%) were diabetic. According to the PSQI score, 66 patients (54\%) were classified as PS and 56 (46\%) as GS. However, 94 patients (77\%) considered their SQ as good or very good.

Distribution of age, gender, associated clinical conditions, medications and laboratory values among GS and PS are summarized in Table 1. Compared to GS, PS were older (66 \pm 14.2 vs $58 \pm 17.0, p=0.004$ ), were more frequently female ( $59 \%$ vs $26 \%, \mathrm{p}=0.001)$ and had a worse renal function $(49 \pm 19.1 \mathrm{vs} 57 \pm 23.2 \mathrm{ml} / \mathrm{min} / 1.73$ $\mathrm{m}^{2}, \mathrm{p}=0.037$ ). There were no differences regarding diabetes, COPD, uric acid, inflammation (CRP) and albuminuria between PS and GS. Albeit not statistically significant, comorbid cardiovascular diseases, as well as depression were somewhat more frequent among PS (Table 1). Iron levels tended to be lower (66 \pm 28.8 vs $78 \pm$ $24.9 \mu \mathrm{g} / \mathrm{ml}$ ) in PS, without significant impact in hemoglobin concentration. We found no differences in calcium, phosphate or parathormone values between the two groups. However, there was a positive correlation between phosphate levels and PSQI score $(r=0.234, p=0.012)$, and vitamin $D$ concentration was significantly lower in PS (17 \pm 7.2 vs $23 \pm 15.1 \mathrm{ng} / \mathrm{ml})$. Multiple logistic regression analysis including all factors that reached nominal significance (age, gender, eGFR, iron, phosphate and vitamin D), revealed female gender (OR 9.929, CI 2.483 $39.705, \mathrm{p}=0.001$ ) as the only independently associated variable. Subsequently, a separate analysis of the data was carried out for male and female patients. Among men, vitamin D and phosphate levels were the only variables that remained statistically different between PS and GS. In contrast, PS women showed worse renal function and iron status than women who were GS, without significant difference in phosphate or vitamin D levels. Inflammation (assessed by CRP levels) was also significantly higher among PS women.

Regarding long term complications and outcome, hospitalization episodes (any cause) were significantly more frequent among PS (64\% vs 44\%), a difference that was more pronounced among men. Despite worse renal function at baseline, GFR decline as well as evolution to end stage renal disease (ESRD) was not significantly different between both groups. Although not statistically significant, mortality rate was somewhat higher in PS (18\% vs $16 \%)$.

\section{Discussion}

Recent studies reported very variable prevalence of poor sleep in CKD (prior to ESRD), from 14\% to as high as 85\% [4]. Most of the participants in our study (54\%) had indicators of poor sleep in the PSQI metrics. Interestingly, their subjective perception of SQ was significantly better than showed by the PSQI test (77\% considered their sleep was good or very good). This discrepancy may help to explain the variable frequency of sleep disturbances found in surveys of CKD patients, if different questionnaires are used to assess SQ.

The aim of this study was to identify factors that may influence SQ in CKD patients, including renal function itself. Most previously reported studies found an association of older age with poor SQ, both in CKD and in the general population [9]. Although this association was also present in our cohort, a multivariate analysis failed to show age as an independent predictive factor. Our study revealed a significant difference in SQ by gender, which is consistent with previous data in the dialysis population [3] [10]. In fact, gender appears to be the single most important factor discerning between PS and GS in our cohort, an effect that was not obvious in previous investigations [9]. This difference in SQ between male and female is not specific for CKD, since it has been largely reported in other cohorts [11] [12]. Possible explanations suggested for the influence of gender in SQ include perception of social support, religious convictions, health-related behaviors and outlook that may vary 
Table 1. Patient characteristics according to sleep quality.

\begin{tabular}{|c|c|c|c|}
\hline & GS & PS & $\mathrm{p}$ \\
\hline N (\%) & $56(46)$ & $66(54)$ & \\
\hline Age (yrs) & $58 \pm 17.0$ & $66 \pm 14.2$ & 0.004 \\
\hline Female (\%) & $14(26)$ & 36 (59) & 0.001 \\
\hline BMI $\left(\mathrm{Kg} / \mathrm{m}^{2}\right)$ & $28.8 \pm 5.15$ & $28.6 \pm 4.12$ & 0.946 \\
\hline Diabetes (\%) & $15(28)$ & $21(34)$ & 0.286 \\
\hline CV event (\%) & $6(11)$ & $10(16)$ & 0.590 \\
\hline COPD (\%) & $4(7)$ & $8(13)$ & 0.373 \\
\hline Depression (\%) & $2(4)$ & $6(10)$ & 0.279 \\
\hline Hemoglobin (g/dl) & $13.7 \pm 1.72$ & $13.2 \pm 1.55$ & 0.109 \\
\hline Creatinine (mg/dl) & $1.5 \pm 0.57$ & $1.5 \pm 0.71$ & 0.619 \\
\hline Initial GFR (ml/min) & $57 \pm 23.2$ & $49 \pm 19.1$ & 0.037 \\
\hline Final GFR (ml/min) & $53 \pm 27.1$ & $46 \pm 24.5$ & 0.160 \\
\hline GFR decline (ml/min) & $-4.3 \pm 11.4$ & $-2.8 \pm 15.1$ & 0.543 \\
\hline Alb/Cr ratio (mg/g) & $273 \pm 556.9$ & $482 \pm 1133.9$ & 0.221 \\
\hline Uric acid (mg/dl) & $6.6 \pm 1.87$ & $6.6 \pm 1.80$ & 0.969 \\
\hline Iron (ug/ml) & $78 \pm 24.9$ & $66 \pm 28.8$ & 0.039 \\
\hline Ferritin (mg/dl) & $126 \pm 117.2$ & $106 \pm 86.8$ & 0.368 \\
\hline Calcium (mg/dl) & $9.6 \pm 0.47$ & $9.6 \pm 0.54$ & 0.899 \\
\hline Phosphate (mg/dl) & $3.4 \pm 0.62$ & $3.6 \pm 0.64$ & 0.117 \\
\hline PTH (pg/ml) & $112 \pm 103.0$ & $140 \pm 143.6$ & 0.383 \\
\hline 25 OH D3 & $23 \pm 15.1$ & $17 \pm 7.2$ & 0.043 \\
\hline PCR (mg/dl) & $0.41 \pm 0.506$ & $0.69 \pm 1.145$ & 0.170 \\
\hline Hospitalization, any cause (\%) & $24(44)$ & $39(64)$ & 0.028 \\
\hline ESRD (\%) & $3(6)$ & $5(8)$ & 0.429 \\
\hline Death, any cause (\%) & $9(17)$ & $11(18)$ & 0.523 \\
\hline
\end{tabular}

GS = Good sleepers according to PSQI; PS = Poor sleepers according to PSQI; CV = cardiovascular; COPD = chronic obstructive pulmonary disease; GFR = Glomerular filtration rate; $\mathrm{Alb}=$ albumin; $\mathrm{Cr}=\mathrm{creatinine} ; \mathrm{PTH}=$ parathormone; PCR = C-reactive protein; ESDR = End stage renal disease.

among both genders in different geographical areas. Other confounding factors, such as education, marital state and employment status may also influence on SQ differences between genders.

Association of SQ and renal function is controversial [9] [13] [14]. We observed a correlation between impairment of sleep and renal function using calculated eGFR (and not serum creatinine) as measure of renal dysfunction. This may be explained by the female predominance of sleep problems and the factoring in of gender in the MDRD-4 formula. In fact, decline in SQ may already start in early stages of renal disease and therefore would not be simply related with the severity of renal impairment [15] [16].

We investigated whether other factors common in CKD—like anemia, diabetes and inflammation — could influence SQ. We did not find association between SQ and anemia, only a somewhat lower iron concentration in poor sleepers that was not clinically relevant. The presence of anemia has been plausibly linked with sleep disturbance in the literature. Except for the case of RLS [5], we speculate whether this might be a spurious association due to the presence of symptoms of anemia like fatigue, tiredness, lack of concentration or daytime sleepiness. Moreover, since hemoglobin values are physiologically lower in women, a gender effect may also bias the comparison of hemoglobin concentration between poor and good sleepers. Previous investigations suggested 
that sleep disorders are more common in diabetic patients [17]. We did not observe significant differences in sleep quality scores between diabetic and non-diabetic patients. This discrepancy with other studies might be due to the low number of diabetics in our cohort who had severe comorbidity-such as cardiovascular problems and neuropathy-since our target population was non-dialysis CKD. Elevated CRP has been associated with increased vascular inflammation due to sleep deprivation-related hypertension [18]. Interestingly, our data suggest an association between inflammation and SQ in female CKD patients that deserves further investigation.

Mineral metabolism disturbances are common in renal disease. Some studies have shown an association between sleep problems and higher levels of phosphate and parathyroid hormone in hemodialysis and CKD patients [9] [19], that may improve after parathyroidectomy [20]. Our results suggest a correlation between PSQI score and phosphate levels, remarkably in males. Patients with poor SQ were also characterized by lower plasmatic vitamin D concentration. As far as we are aware, this is the first report underlining the association between low vitamin D levels and poor SQ in renal disease. In agreement with this observation, recent data support an inverse relationship between vitamin D levels and OSA severity [21]. Furthermore, vitamin D supplementation has been linked with RLS improvement [22]. Since both conditions (OSA and RLS) are common in $\mathrm{CKD}$, it is plausible that vitamin D deficiency plays a role in sleep disturbance in renal disease.

Another finding of our study was that hospitalization rate (for any cause) in CKD patients increases markedly with the decrease of sleep quality. Poor sleep quality has been associated with an increased risk of mortality in pre-ESRD and hemodialysis patients [3] [14]. We did not find a significant correlation between SQ and mortality, perhaps due to the fact that our study population has less cardiovascular comorbid conditions than patients on dialysis or pre-dialysis. Poor sleep quality has also been related with the progression of cardiovascular disease and renal damage [4] [23]. In contrast, we did not detect an association between renal function decline and SQ. Despite progression of cardiovascular disease, sleep disturbances may start in the early stages of renal disease and thus would have less impact in renal outcome [15] [16].

\section{Conclusion}

In summary, this study shows that most non-dialysis CKD patients have poor sleep quality, especially women. Poor sleep is associated with worse renal function, higher phosphate and lower vitamin D levels. Sleep disturbance was also a predictor of higher hospitalization rate in our population. A limitation of our study is the use of PSQI as only measurement of sleep quality. Since a comprehensive polysomnography was not performed, it is not possible to ascertain the exact type of sleep disturbance and sleep pattern in our patients. There is no standardized consensus on criteria for subjective reporting of sleep quality. Global PSQI Scores above 5 resulted in a sensitivity of $98.7 \%$ and specificity of $84.4 \%$ to detect sleep disturbances [24]. Alcohol intake, smoking, medications and other factors that may have an impact on sleep were not evaluated. Besides renal function and other health and physical conditions, sleep is under the influence of habits and behaviors, culture, and even economic and political, thus hampering inter-study comparisons. Further large-scale, multi-center studies are needed to better address this problem.

\section{References}

[1] Pierratos, A. and Hanly, P.J. (2011) Sleep Disorders over the Full Range of Chronic Kidney Disease. Blood Purification, 31, 146-150. http://dx.doi.org/10.1159/000321859

[2] Mucsi, I., Molnar, M.Z., Ambrus, C., et al. (2005) Restless Legs Syndrome, Insomnia and Quality of Life in Patients on Maintenance Dialysis. Nephrology Dialysis Transplantation, 20, 571-577. http://dx.doi.org/10.1093/ndt/gfh654

[3] Elder SJ, Pisoni RL, Akizawa T, et al. (2008) Sleep Quality Predicts Quality of Life and Mortality Risk in Hemodialysis Patients: Results from the Dialysis Outcomes and Practice Patterns Study (DOPPS). Nephrology Dialysis Transplantation, 23, 998-1004. http://dx.doi.org/10.1093/ndt/gfm630

[4] Turek, N.F., Ricardo, A.C. and Lash, J.P. (2012) Sleep Disturbances as Nontraditional Risk Factors for Development and Progression of CKD: Review of the Evidence. American Journal of Kidney Diseases, 60, 823-833. http://dx.doi.org/10.1053/j.ajkd.2012.04.027

[5] Calviño, J., Cigarran, S., Lopez, L.M., Martinez, A. and Sobrido, M.J. (2015) Restless Legs Syndrome in Non-Dialysis Renal Patients: Is It Really That Common? Journal of Clinical Sleep Medicine, 11, 57-60.

[6] Buysse, D.J., Reynolds 3rd, C.F., Monk, T.H., Berman, S.R. and Kupfer, D.J. (1989) The Pittsburgh Sleep Quality Index: A New Instrument for Psychiatric Practice and Research. Psychiatry Research, 28, 193-213.

http://dx.doi.org/10.1016/0165-1781(89)90047-4 
[7] Levey, A.S., Bosch, J.P., Lewis, J.B., Greene, T., Rogers, N. and Roth, D. (1999) A More Accurate Method to Estimate Glomerular Filtration Rate from Serum Creatinine: A New Prediction Equation. Modification of Diet in Renal Disease Study Group. Annals of Internal Medicine, 130, 461-470. http://dx.doi.org/10.7326/0003-4819-130-6-199903160-00002

[8] National Kidney Foundation (2002) K/DOQI Clinical Practice Guidelines for Chronic Kidney Disease: Evaluation, Classification, and Stratification. American Journal of Kidney Diseases, 39, S1-266.

[9] Zhang, J., Wang, C., Gong, W., et al. (2014) Association between Sleep Quality and Cardiovascular Damage in Pre-Dialysis Patients with Chronic Kidney Disease. BMC Nephrology, 15, 131. http://dx.doi.org/10.1186/1471-2369-15-131

[10] Tel, H. (2009) Determining Quality of Life and Sleep in Hemodialysis Patients. Dialysis \& Transplantation, 38, 210215. http://dx.doi.org/10.1002/dat.20296

[11] Grandner, M.A., Patel, N.P., Gehrman, P.R., Xie, D., Sha, D., Weaver, T. and Gooneratne, N. (2010) Who Gets the Best Sleep? Ethnic and Socioeconomic Factors Related to Sleep Complaints. Sleep Medicine, 11, 470-478. http://dx.doi.org/10.1016/j.sleep.2009.10.006

[12] Arber, S., Bote, M. and Meadows, R. (2009) Gender and Socio-Economic Patterning of Self-Reported Sleep Problems in Britain. Social Science \& Medicine, 68, 281-289. http://dx.doi.org/10.1016/j.socscimed.2008.10.016

[13] Kurella, M., Luan, J., Lash, J.P. and Chertow, G.M. (2005) Self-Assessed Sleep Quality in Chronic Kidney Disease. International Urology and Nephrology, 37, 159-165. http://dx.doi.org/10.1007/s11255-004-4654-Z

[14] Kumar, B., Tilea, A., Gillespie, B.W., et al. (2010) Significance of Self-Reported Sleep Quality (SQ) in Chronic Kidney Disease (CKD): The Renal Research Institute (RRI)-CKD Study. Clinical Nephrology, 73, 104-114. http://dx.doi.org/10.5414/CNP73104

[15] De Santo, R.M., Bartiromo, M., Cesare, C.M. and Cirillo, M. (2008) Sleep Disorders Occur Very Early in Chronic Kidney Disease. Journal of Nephrology, 21, S59-S65.

[16] Iliescu, E.A., Yeates, K.E. and Holland, D.C. (2004) Quality of Sleep in Patients with Chronic Kidney Disease. Nephrology Dialysis Transplantation, 19, 95-99. http://dx.doi.org/10.1093/ndt/gfg423

[17] Kutner, N.G., Zhang, R., Huang, Y. and Bliwise, D.L. (2007) Association of Sleep Difficulty with Kidney Disease Quality of Life Cognitive Function Score Reported by Patients Who Recently Started Dialysis. Clinical Journal of the American Society of Nephrology, 2, 284-289. http://dx.doi.org/10.2215/CJN.03000906

[18] Desideri, G., Groce, G. and Ferri, C. (2004) Does Nondipping Blood Pressure Profile Contribute to Vascular Inflammation during Sleep Deprivation? Journal of the American College of Cardiology, 44, 1529-1530. http://dx.doi.org/10.1016/j.jacc.2004.07.008

[19] De Santo, R.M., Esposito, M.G., Cesare, C.M., et al. (2008) High Prevalence of Sleep Disorders in Hemo-Dialyzed Patients Requiring Parathyroidectomy. Journal of Renal Nutrition, 18, 52-55. http://dx.doi.org/10.1053/j.jrn.2007.10.011

[20] Esposito, M.G., Cesare, C.M., De Santo, R.M., et al. (2008) Parathyroidectomy Improves the Quality of Sleep in Maintenance Hemodialysis Patients with Severe Hyperparathyroidism. Journal of Nephrology, 21, S92-S96.

[21] Kerley, C.P., Hutchinson, K., Bolger, K., McGowan, A., Faul, J. and Cormican, L. (2016) Serum Vitamin D Is Significantly Inversely Associated with Disease Severity in Caucasian Adults with Obstructive Sleep Apnea Syndrome: A Case Control Study. Sleep, 39, 293-300.

[22] Wali, S., Shukr, A., Boudal, A., Alsaiari, A. and Krayem, A. (2015) The Effect of Vitamin D Supplements on the Severity of Restless Legs Syndrome. Sleep and Breathing, 19, 579-583. http://dx.doi.org/10.1007/s11325-014-1049-y

[23] Hoevenaar-Blom, M.P., Spijkerman, A.M., Kromhout, D., van den Berg, J.F. and Verschuren, W.M. (2011) Sleep Duration and Sleep Quality in Relation to 12-Year Cardiovascular Disease Incidence: The MORGEN Study. Sleep, 34, 1487-1492. http://dx.doi.org/10.5665/sleep.1382

[24] Backhaus, J., Junghanns, K., Broocks, A., Riemann, D. and Hohagen, F. (2002) Test-Retest Reliability and Validity of the Pittsburgh Sleep Quality Index in Primary Insomnia. Journal of Psychosomatic Research, 53, 737-740.

http://dx.doi.org/10.1016/S0022-3999(02)00330-6 


\section{Submit or recommend next manuscript to SCIRP and we will provide best service for you:}

Accepting pre-submission inquiries through Email, Facebook, LinkedIn, Twitter, etc.

A wide selection of journals (inclusive of 9 subjects, more than 200 journals)

Providing 24-hour high-quality service

User-friendly online submission system

Fair and swift peer-review system

Efficient typesetting and proofreading procedure

Display of the result of downloads and visits, as well as the number of cited articles

Maximum dissemination of your research work

Submit your manuscript at: http://papersubmission.scirp.org/ 